\title{
A dilemma that underlies an existence proof in geometry
}

\section{Carmen Samper, Patricia Perry, Leonor Camargo, Adalira Sáenz-Ludlow \& Óscar Molina}

\section{Educational Studies in Mathematics} An International Journal

ISSN 0013-1954

Volume 93

Number 1

Educ Stud Math (2016) 93:35-50

DOI 10.1007/s10649-016-9683-x

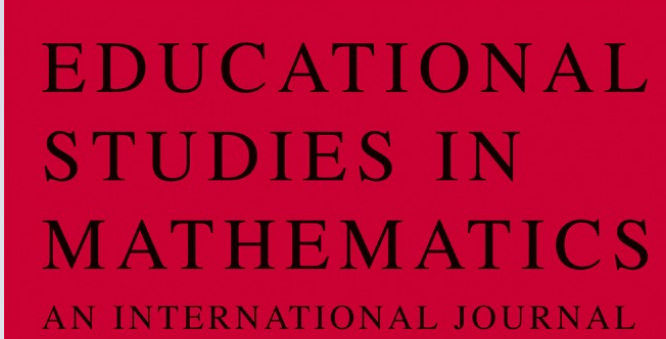

EDITOR-IN-CHIEF : MERRILYN GOOS

Volume 93 No. 1 September 2016

을 Springer 


\title{
A dilemma that underlies an existence proof in geometry
}

\author{
Carmen Samper $^{1} \cdot$ Patricia Perry $^{2} \cdot$ Leonor Camargo $^{3}$. \\ Adalira Sáenz-Ludlow ${ }^{4}$. Óscar Molina ${ }^{5}$
}

Published online: 6 February 2016

(C) Springer Science+Business Media Dordrecht 2016

\begin{abstract}
Proving an existence theorem is less intuitive than proving other theorems. This article presents a semiotic analysis of significant fragments of classroom meaning-making which took place during the class-session in which the existence of the midpoint of a linesegment was proven. The purpose of the analysis is twofold. First follow the evolution of students' conceptualization when constructing a geometric object that has to satisfy two conditions to guarantee its existence within the Euclidean geometric system. An object must be created satisfying one condition that should lead to the fulfillment of the other. Since the construction is not intuitive it generates a dilemma as to which condition can be validly assigned initially. Usually, the students' spontaneous procedure is to force the conditions on a randomly chosen object. Thus, the second goal is to highlight the need for the teacher's mediation so the students understand the strategy to prove existence theorems. In the analysis, we use a model of conceptualization and interpretation based on the Peircean triadic SIGN.
\end{abstract}

Carmen Samper

csamper@pedagogica.edu.co

Patricia Perry

pperryc@yahoo.com.mx

Leonor Camargo

lcamargo@pedagogica.edu.co

Adalira Sáenz-Ludlow

sae@uncc.edu

Óscar Molina

oscarjmolina@gmail.com

1 Universidad Pedagógica Nacional, Tv. 2A No. 67-71, Bogotá, Colombia

2 Universidad Pedagógica Nacional, Cra. 45A No. 123-64, Bogotá, Colombia

3 Universidad Pedagógica Nacional, Carrera 68A No. 22A-75 casa 7, Bogotá, Colombia

4 University of North Carolina at Charlotte, 1550 Daybreak Ridge, Kannapolis, NC 28081, USA

5 Universidad Pedagógica Nacional, Calle 188 No. 55-69, int 3 apt. 501, Bogotá, Colombia 
Keywords Existence proofs · Students' procedure to prove existence theorems $\cdot$ Meaningmaking $\cdot$ Teacher semiotic-mediation

Throughout the last 10 years, the setting for our research project has been the geometry courses for the pre-service high-school-teacher program offered by Universidad Pedagógica Nacional (Colombia), an institution that prepares K-12 teachers. The central emphasis of our research is the improvement of teaching-learning. Recognizing proving as a fundamental process necessary for the development of geometric reasoning, we have progressively developed and refined a geometry curriculum in which both conjecturing and conjectures, proving and proofs are the organizing themes (Samper, Camargo, Molina, \& Perry, 2013).

As Radford, Schubring, and Seeger (2011) stress, teaching and learning mathematics primarily consists of enunciating and interpreting signs, and meanings evolve within social practices where verbal and non-verbal signs are produced. Therefore, they claim, any study of these processes should be from a semiotic perspective, because it provides the necessary theory and methodological tools. This paper analyzes a classroom-episode to illustrate the studentteachers' semiotic process through which they constructed meaning and made sense of the logical strategy to prove the Midpoint Existence Theorem. ${ }^{1}$ It is important to understand geometric definitions carry with them only potential existence rather than actual existence. In elementary geometry, proving an existence theorem sometimes requires the initial construction of a geometric object with some, not all, of the properties included in the definition. One of the fundamental difficulties encountered in the proof is knowing which properties to assign first. These should be only those indispensable to infer-and-justify the remaining properties, using known elements of the theoretical system.

In our teaching-experience, we have repeatedly noticed that students spontaneously use the following procedure to prove the existence of a geometric object. They Randomly Choose an object and then Force it to satisfy the needed Properties (RCFP). ${ }^{2}$ Therefore, a didactical intervention is required to help them understand the progressive construction of the object in a proof: initially assign exactly those properties that permit justifying the rest. This intervention should involve the students' interactive and genuine participation to make sense of why the above procedure works. When the course requirement is to generate ideas for producing a proof, students construct meanings and experience proving as a process which involves their analytic potential. When the teacher enunciates a theorem and reproduces a proof for the students, it becomes a memorization exercise.

\section{Literature review}

Researchers have pointed out difficulties that hinder the process of producing a proof; they are many and different in nature. Related to the difficulty we want to expose is, as Weber (2001) mentions, the students' lack of "strategic knowledge" or knowing what mathematical fact or method to use in a proof. Selden and Selden (2011), and Perry, Camargo, Samper, and Rojas (2006) refer to specific aspects of the strategic knowledge. Selden and Selden include: (i) deciding between choosing an element that belongs to a certain set or constructing the element,

\footnotetext{
${ }^{1}$ Given $\overline{A B}$, there exists a point $M$ that is midpoint of the segment.

${ }^{2}$ We will use the acronym RCFP to denote the mentioned action.
} 
(ii) having to structure a proof of a theorem whose thesis is a disjunction. Perry et al. (2006) mention: (iii) differentiating between localizing, choosing and determining an element, and (iv) understanding the logic of connectives and the tautologies associated with each connective. The RCFP occurrence links (i) and (iii) since the students do not construct a geometric element with special properties but, instead, decide to choose it randomly and then force upon it the properties that allow fulfilling what is needed in the proof. Proving the existence of an object, defined through a conjunction of properties has something to do with (ii) and (iv), because some properties must be assigned originally to an element and the others deduced from them.

Related to the specific topic of this paper, de Guzmán, Hodgson, Robert, and Villani (1998) point out various reasons why existence proofs are particularly difficult for tertiary students: they do not see the need for proving that something exists; it's contrary to their experience with high-school mathematics where the existence of objects involved in a problem is assumed; it's hard to imagine the specific mathematical object that must be constructed - this is a creative conceptual act. Therefore, students need to learn special procedures to produce existence proofs. In some cases, the Axiom of Choice is used (e.g., proving that there exists a basis for the real numbers as a vector space over the rational numbers); in others, the proof is by contradiction (e.g., proving there exist an infinite number of prime numbers). In elementary geometry, the procedure is as described in the Introduction.

As Lannin, Barker, and Townsend (2007) mention, it's worthwhile to use student errors as a didactical mean to promote learning. This is possible only if the teacher understands the conceptual issue that underlies such behavior. Precisely, due to our thorough analysis of the semiotic process of the students' meaning-making of the Midpoint-Existence-Theorem proof, we were able to hypothesize about what the nature of RCFP could be.

\section{Theoretical elements}

A month after the semester started, the students, in the plane-geometry course, had to prove the existence of the midpoint of a segment. The teacher involved them in a dialogue to aid comprehension of the procedure for this type of proof. The interaction between teacher and students and the students' progressive understanding is analyzed using Sáenz-Ludlow and Zellweger's (2012) model of interpretation, which is rooted in the Peircean triadic SIGN. ${ }^{3} \mathrm{We}$ considered their proposal relevant because we believe that meaning-making is a dialogical process; the model offers analytic richness and the means to study the process in detail. Its use forced us to observe not only what is verbalized but also to explore how to interpret what is said; not only to consider consecutive specific verbalizations but also the complete cycle in which new conceptions, tending towards the mathematical concept in question, are generated, regenerated and refined. The model breaks down the complexity of a communicative act, permitting a thorough analysis of the meaning-making act, through the different types of objects and signs that must be identified.

\footnotetext{
${ }^{3}$ The word SIGN (in upper case letters) stands for the unified and undividable relation among the three components of the Peircean "sign". In their model, SIGN is notated by the triplet sign-object, sign-vehicle, sign-interpretant.
} 


\subsection{A model of classroom interpretation}

Charles S. Peirce's distinctive contribution is having conceived SIGN as a holistic entity with three components: sign-object, that which is alluded to in a communication or thought; signvehicle, the representation with which the object is alluded to (e.g., a word, gesture, graph or a combination of these); and sign-interpretant, that which is produced by the sign-vehicle in the mind of whoever perceives and interprets it. There is semiotic activity whenever two or more people engage in communication activity; such activity interprets SIGNS from different semiotics systems (linguistic, gestural, mathematical, etc.).

Sáenz-Ludlow and Zellweger's model conceptualizes classroom interpretation as a progressive, ever changing signifying process. During this process, a person not only inter-acts with other people (Self-Others or Inter) but also co-acts with the Self (Self-Self or Intra). We shall use the expression inter-intra to denote both actions: with oneself and with others. These interpretations lead to the emergence and refining of inter-intra cycles of objectification, which consist of decoding, abstracting and encoding actions, that follow from intentionally constructed and highly coordinated sign-interpretant formations.

The model looks upon the teaching-learning of mathematics as a semiotic process of interpretation that takes place within the socio-mathematical semiotic reality that teachers and students inherit and jointly activate in the classroom. It suggests that, during interpretation, the formation of students' mathematical conceptions and their approximation to mathematical concepts are obtained with the teacher's guidance, following a progressive process of interintra interpretation. In addition, it emphasizes that the teacher's awareness of the evolving nature and refinement of his own process of interpretation and, especially, that of the students is essential to maintain a collaborative and dynamic teaching-learning signifying practice that engages the students' subjectivity in their meaning-making processes.

\subsection{Semiotic activity in the construction of a mathematical object}

The model describes the semiosis that takes place in a verbal exchange constituted by two turns between persons $\mathrm{X}$ and $\mathrm{Y}$, in this case, teacher and student. In an intra-interpretation act, $\mathrm{Y}$ selects a particular aspect of a sign-object that is part of his sign-interpretant, encodes it and expresses it in a sign-vehicle addressed to $\mathrm{X}$. In an inter-interpretation act, that takes place within his knowledge and experience, $\mathrm{X}$ decodes the sign-vehicle emitted by $\mathrm{Y}$ and constructs a sign-interpretant which determines a sign-object that can be in lesser or greater consonance with the one $\mathrm{Y}$ intends to communicate. In turn, $\mathrm{X}$ chooses an aspect of his newly constructed sign-object and the process is repeated, $\mathrm{X}$ being emitter and $\mathrm{Y}$ receiver.

Which sign-object does the emitter select and which does the receiver interpret in a mathematics class? Which object are they referring to? Peirce's genius differentiates three subcategories of sign-objects, in this case: Real Mathematical Object (RMO), immediateobject and dynamic-object. Our focus is the $R M O$, a historic-cultural object (e.g., a mathematical concept or procedure) constructed by the community of mathematicians, that remains unchanged in the teaching-learning activity. The immediate-object, constituted by specific aspects of the $R M O$, is encoded in the sender's sign-vehicle while the dynamic-object (aspects decoded) is generated in the receiver's sign-interpretant.

Our analysis focuses on the sign-vehicles used by teacher and students to convey their immediate sign-objects, the sign-interpretants these sign-vehicles produce in the minds of the receivers (teacher or students), and the dynamic-objects these sign-interpretants generate. 
Through a thorough and intentional interpretation of sign-vehicles, as Peirce proposes, signinterpretants and dynamic-objects are inferred.

\subsection{Meaning-making in the classroom and the teacher semiotic mediation}

When trying to make sense of an $R M O$ in the classroom, there is a dialogic interaction. The individuals that engage in a conversation have different levels of knowledge, with respect to the $R M O$, and different goals. The teacher's goal is to support the students' meaning-making of the $R M O$; the students' goal is to genuinely participate in the process, interpreting the teacher's sign-vehicles, to meaningfully construct the $R M O$. Their emerging dynamic-objects will agree, to a lesser or greater degree, with the teacher's intended immediate-object.

In an interpretation act, the teacher must contemplate the $R M O$ from two perspectives. From the mathematical perspective since he/she evokes meanings of certain aspects of the $R M O$ to use them as a reference for specific actions, and from the didactical perspective because the mathematical object being constructed emerges from intentional teacher and student actions as they interleave their goals. The teacher's actions are influenced by beliefs, knowledge, and previous experiences. Therefore, his/her interpretants and dynamic-objects are more of a didactic nature.

The teacher's intentional semiotic mediation is constituted by deliberate actions to facilitate and guide the convergence of the students' evolving dynamic-objects to his/her intended immediate-object. These mediating actions take into account his/her interpretations of the students' dynamic-objects, particular aspects of the RMO. The integration of the most significant mathematical aspects of what the teacher believes are the students' dynamic-objects with didactical considerations, such as identifying aspects of the $R M O$ on which he/she must focus to increase student meaning-making and whether it is developing in an acceptable manner, emerges in a dynamic-object of his/her own. The teacher then generates sign-vehicles that he/ she deems necessary to facilitate the evolution of the students' dynamic-objects. We denominate this emerging and evolving teacher dynamic- object as a didactical dynamic-object (Tddo) (Perry, Camargo, Samper, Sáenz-Ludlow, \& Molina, 2014).

The following example illustrates the above:

\subsection{Proof of the Midpoint-Existence-Theorem}

In the introduction, we mentioned the general procedure used to prove the existence of an object whose definition includes two or more conditions. In the case of the MidpointExistence-Theorem, a segment point that is equidistant to its endpoints has to be constructed. The theoretic system constituted during the plane-geometry course is based on Birkhoff's model (1932) for Euclidean geometry in which the facts, embodied in a ruler and protractor, are introduced.

Specifically, the Line-Real Numbers-Postulate sets up a bijective correspondence between the real numbers and the points of a line. ${ }^{4}$ This postulate is only used to prove relationships between points on a line and doesn't lead to coordinate geometry. Creating a point that satisfies equidistance is achieved by: (1) creating the line determined by the endpoints of the segment,

\footnotetext{
${ }^{4}$ Given a line, there's a correspondence between the points of the line and the real numbers such that: (i) to each point there corresponds exactly one real number; (ii) to each real number there corresponds exactly one point of the line. The number assigned to point $A$ is called its coordinate and represented by $\mathrm{c}(A)$.
} 
for example $A$ and $B$; (2) assigning coordinates $z$ and $y$ to these points (Line-Real NumbersPostulate (i)), (3) finding $w$ the average of the coordinates, and (4) assigning to $w$ the corresponding point $M$ of the line (Line-Real Numbers-Postulate (ii)). Proving $M$ is actually between $A$ and $B$ (Betweeness Definition ${ }^{5}$ ) is a direct consequence of steps (1) through (4). At this point, the theoretical system the students could use included: midpoint, segment and betweeness definitions, Line-Real Numbers-Postulate, and theorems about betweeness such as Double Order-Betweeness-Theorem, ${ }^{6}$ Point-Between-Theorem ${ }^{7}$ and Point-on-a-SideTheorem. ${ }^{8}$

\section{Research methodology}

\subsection{Research setting}

A main goal of the course, from which the analyzed episode is taken, is that students learn to prove deductively, while gradually configuring a theoretic system in which definitions, postulates and theorems are introduced when needed to solve a problem, the latter once they have been proven. The methodological teaching approach rests upon three elements: mathematical tasks, social interaction in class, and the use of dynamic geometry (Perry, Samper, Camargo, \& Molina, 2013). The mathematical tasks are mainly open-conjecturing-problems, in the sense of Baccaglini-Frank and Mariotti (2010). Students can explore using dynamic geometry to find regularities, formulate conjectures, and validate these within the theoretic system constituted up to that point. Social interaction is the norm in the course because meaning-making as a collective enterprise is one of our main underlying research hypotheses. Students can communicate ideas, accept or reject those of others with valid arguments, participate in the formulation of theorems and definitions, and collectively construct proofs. The third element is the use of dynamic geometry as a mediating tool for learning. As Mariotti (2009) points out, the principal characteristic of the "signs" derived from the use of dynamic geometry is their relation with the actions students do with them. These actions are paramount for the semiotic activity that takes place in the classroom. The ideas that arise during the use of dynamic geometry are socially shared and thus can evolve towards mathematical SIGNS related to the $R M O$, focus of each class.

The class met twice a week for $2 \mathrm{~h}$ sessions. The 14 students, aged between 18 and 24, were organized in small work groups to favor interaction. They could use computers with the Cabri software incorporated. Teacher and students kept an active participatory interaction. The teacher, one of the authors of this article, had previously taught the course at least six times. Therefore, his didactical decisions were based on the conceptual and practical knowledge obtained from these experiences.

When the Midpoint-Existence-Theorem was undertaken, the existence of segments and rays had been proven. These proofs differ from the one at hand; their existence is assured because their definitions mention sets with at least two elements - the points that determine each object. The proofs of the Point-Between-Theorem and the Point-on-a-Side-Theorem,

\footnotetext{
${ }^{5}$ Point $B$ is between points $A$ and $C$ if: (i) $A, B$ and $C$ are collinear and (ii) $A B+B C=A C$.

${ }^{6}$ Given three points $A, B$ and $C$ of line $m$, if $\mathrm{c}(A)<\mathrm{c}(B)<\mathrm{c}(C)$ or $\mathrm{c}(C)$ or $\mathrm{c}(C)<\mathrm{c}(B)<\mathrm{c}(A)$, then $B$ is between $A$ and $C$.

${ }_{8}^{7}$ Given two points $A$ and $B$, there exists a point $C$ between them.

${ }^{8}$ Given two points $A$ and $B$, there exists a point $C$ such that $B$ is between $A$ and $C$.
} 
which also refer to the existence of points, required the use of the Line-Real-NumbersPostulate. This is the first time that students have to face the task of proving the existence of an object (midpoint) that is defined via two properties.

\subsection{Experimental data}

The episode, object of the analysis presented here, was consolidated by reviewing the video and audio recordings. The study of the transcription was focused on the identification of central aspects that hampered meaning-making of the procedure to prove the MidpointExistence-Theorem (RMO). During the retrospective and recursive analysis of the transcript, we were able to isolate six significant meaning-making moments that can be viewed as a succession of interpreting cycles. They enclose the construction of different sign-objects related to an aspect of the $R M O$.

In the analysis, we might group together various student utterances because, as a whole, they make up a complete cycle of meaning-making. Similarly, two or more teacher interventions could be taken as only one sign-vehicle because some add more detail to the immediate sign-object that the teacher is trying to communicate.

\section{Analysis}

\subsection{Semiotic interaction}

When presenting the analysis, an intervention of a classroom participant is symbolized by a bracket that contains a number and the first letter of the participant's pseudonym. For example, [28Á] indicates that Ángela's sign-vehicle is the 28th intervention in the transcription. The purpose is to convey a sense of participation, in real time, and identify the student. The absence of certain lines indicates non-significant detours in the students' ideas.

Each moment shows essential aspects of the construction of the existence proof of the midpoint, and illustrates the teacher's didactical semiotic mediation. This mediation is didactical because the teacher considered not only the $R M O$, as he interpreted it, but also student constructed meanings during their collaborative participation. These meanings were the results of students' constructions of interpretants and dynamic-objects, as inferred by the researchers, and the ensuing immediate sign-objects encoded in their sign-vehicles (complete and incomplete sentences) according to their ongoing understanding. An example of the analysis carried out can be seen in Table 1 .

The retrospective synthesis of teacher and students inter-intra interpretation, as they engaged in proving the existence of the midpoint, is synthesized in Fig. 1; Section 4.2. The mediation can be traced in terms of the inferred didactical dynamic-objects of the teacher $(\mathrm{T} d d o$ ) and the inferred dynamic-objects of the students ( $\mathrm{S} d o$ ) that emerged prompted by the teacher's intentional, conceptual, and practical actions, encoded in verbal sign-vehicles or in iconic sign-vehicles of visual nature.

To begin the class, the teacher reminded the students of the Midpoint Definition"; the task now was to prove its existence. As always, he first enunciated the geometric statement: "We are going to justify that if we have a segment $A B$, then there exists exactly one midpoint". He

${ }^{9} M$ is the midpoint of $\overline{A B}$ if (i) $M, A$ and $B$ are collinear and (ii) $A M=M B$. 
Table 1 Example semiotic mediation in classroom
Sign-vehicle
Analysis

T: $\quad$ How can we define midpoint of $\overline{A B}$ ?

RMO: Midpoint definition

S: $\quad$ It is a point $M$ such that $A M=B M$. ( $A M$ denotes the distance from $A$ to $M$.)

Sign-interpretant: could be mental iconic representation of a segment and its midpoint, visually satisfying betweeness and equidistance

Dynamic-object: at least equidistance property of midpoint Immediate-object: midpoint definition

T: (Drawing $\overline{A B}$ and a point above it that seems to be equidistant from $\mathrm{A}$ and $\mathrm{B}$ )

Immediate-object: existence of many points that satisfy equidistance to endpoints of segment

Is this point the midpoint of $\overline{A B}$ ?

Sign-interpretant: the two properties required to be a midpoint: betweeness and equidistance

Didactic-dynamic-object: necessity of realization that betweeness property must be part of the definition

set up a proof-frame (Table 2), on the board, to record the sequential emergence of each assertion (first column of a row) and the corresponding warrant(s) and data (second column of the same row). Each assertion was collectively agreed upon.

Moment 1: First step of proof is choosing a point between $A$ and $B$. To encourage the coconstruction of the proof, the teacher asked Laura to produce the first step. Laura and Ángela stated their ideas using verbal statements, which constitute their sign-vehicle: "say that there exists a point in the midst of $A$ and $B$ " [8L],"there has to be a point between $A$ and $B$ " [10Á]. Laura and Ángela completed their sign-vehicle when the teacher asked how to document their proposal in the frame: "With the Point-Between-Theorem" [14L]; "Let $X$ be a point between $A$ and $B \ldots$ such that $A, X, B$ " [21Á, 23Á], which is how students and teacher communicate that $X$ is a point between $A$ and $B$. In [14L], Laura delivered the theoretical element that justifies their suggestion and, in her interventions [21Á, 23Á], Ángela stated the thesis of such element. Following their instructions, the teacher filled in the second row of the frame (Table 2).

From the words we placed in boldface, we infer that the students' sign-interpretant could include a mental iconic representation of a segment with its midpoint, and possibly the two conditions stated in the midpoint definition: betweeness and equidistance to the segment endpoints. That is, they could have "seen" these in their mental representation. From our perspective, the inferred dynamic-object ( $\mathrm{S} d o 1$, first student dynamic-object) can at least include the betweeness property of the midpoint. Their immediate-object is the complete argument of what they think is the first step of the proof. Alluding to the theorem that allows choosing points between two given points, it is now clear that Laura's expression "in the midst of" refers only to the betweeness property, and that they are not assigning another special property to that point. Therefore, they are convinced that the proof must start by creating a point that satisfies betweeness.

Table 2 First two entries in the proof of Midpoint Existence Theorem

\begin{tabular}{ll}
\hline Assertion & Warrant and data \\
\hline $1 . \overline{A B}$ & Given \\
2. Let $X$ be a point between $A$ and $B$. & Point-Between-Theorem (1) \\
\hline
\end{tabular}


The teacher's sign-vehicle "How do I write that?" [13T, 17T], "Why do you think we can use that theorem?" [27T], communicated his immediate-object, an explanation of why their proposal should be the first step of the proof. The teacher could be thinking that if he provides the opportunity to informally express the idea behind their proposal, the inapplicability of choosing any point would be revealed (Tddo1, first teacher didactical dynamic-object). The teacher's sign-interpretant seems to include what the first step of the proof should be and that the students' suggested step would not lead to the other property: equidistance to the endpoints.

As a response, the students jointly constructed a sign-vehicle, which included gestures: "Because we need a point that is between these two" (Ángela extended thumb and index fingers of her right hand to represent the separation between the endpoints of the segment and moved it horizontally with extended fingers as if covering the segment); "to say... this point... [the midpoint] exists" [28Á] and "To find the midpoint. To say that $X$ is in the midst of $A$ and $B$ " [29L]. From the students' sign-vehicle, we infer that their dynamic and immediate objects have not changed significantly.

Moment 2: Is there a problem with the suggested proof-step? Having registered the argument in the proof-frame, the teacher's sign-vehicle included not only a verbal expression but also an illustration: "What are the two conditions we have to talk about midpoint? [Various students gave the correct answer.] There should be betweeness and equality of distances. With what Laura and Ángela want to do, we would have part of what we need. But I want you (looking at all the students) to tell me if that way of doing the proof is correct or not. Do you agree with what they propose or do you see a little problem with it?" [33T], and "In graphic representation terms, what we have so far is something like this (on the blackboard he drew a segment with $A$ and $B$ as endpoints) and they propose the existence of a point $X$ that satisfies the betweeness relation $A, X, B$ " [41T].

Having confirmed that the students favor the betweeness property initially, we infer that the teacher's sign-interpretant of the students' sign-vehicle includes the hypothesis that the students are going to force the randomly chosen point to satisfy equidistance (RCFP). This could be why he wanted them to remember the midpoint definition. The teacher's hypothesis seems natural due to his experience teaching this course. The frequency of the RCFP procedure in students' proposals had led him to discuss, in the previous class, why it was not an adequate procedure. The teacher's sign-interpretant could also include the idea that illustrating their proposed procedure with a graphic representation would help them realize the problem he is alluding to.

We identify betweeness as a condition for the existence of the midpoint as his immediateobject, and the need to establish what else is required as his didactical-dynamic-object (Tddo2). Specifically, he could be thinking that considering the equidistance property and asking for a revision of the students' proposal (using the segment drawn) would make everyone realize the conflict that emerges when trying to attain the required distance condition.

Laura and Ángela seemed to interpret the teacher's sign-vehicle as unacceptability of their argument because it does not have the formalism he usually requires; so they added other details to it. "Well, teacher, I do not know if it is necessary to say that $A$ and $B$ [exist]; that is, I don't know if that is given because the segment is given or if we have to say it." [37L] and "That $X$ belongs to the segment." [42Á]. Their immediate-object consists of the theoretical elements that could fill in the gap between the first and second step of the proof. Their dynamic-object ( $\mathrm{Sdo2}$ ) could be the conditions stated in the hypothesis of the Point-BetweenTheorem to insure its use in the second step of the proof. The students' immediate-object partially coincides with the teacher's immediate-object in so far as they perceive the teacher's 
dissatisfaction with their suggestion, and they react by providing the details they consider are missing. Yet, what the teacher intended them to realize was that the point they propose (i.e., any point between the endpoints of the segment) will not necessarily satisfy the desired equidistance property.

Moment 3: The problem is how X appears. The teacher kept insisting on the need to revise the students' proposal: "I would like to know if you see something strange, or if we should continue. If we all agree, we will continue." [46T] At that moment, another student, María, intervened. Her initial sign-vehicle was a timid statement "It's that the $X$ appears like... as free" [48M]. Laura and Ángela agreed with María, through a verbal sign-vehicle "Yes, just like that" [49L], and Ángela's nod. The teacher picked up María's idea. His sign-vehicle was composed taking into account the previous interventions: "It's around there... it just appears. Right, María? And when it appears, where can that point appear between $A$ and $B$ ?" [51T]. María answered: "Anywhere in the segment." [52M]. The teacher completed his sign-vehicle, referring to the graphic representation on the blackboard. "Anywhere in the segment. So, what if $X$ appears about here (on the segment representation, he marked a point very close to $A$ )? Or if $X$ appears around here (he marked another point)" [53T].

María interpreted the teacher's request to examine the proposal as an objection to its content; her sign-interpretant seems to include the issue associated with RCFP and that the midpoint has a specific position on the segment. We interpret María's use of the word "free" as the freedom of placing point $X$ anywhere in the segment, something she later reinforces with the use of the words we placed in boldface [48-52M]. This is her immediate-object. Her dynamic-object ( $\mathrm{S} d o 3$ ) could be that the problem the teacher insinuates is related to the way the point appears. María's dynamic-object coincides with the teacher's immediate-object. Laura and Ángela reiterated that they just wanted to create a point between $A$ and $B$ (immediate-object). Although their dynamic object should have been slightly modified by what has been said, it seems to be close to their first dynamic-object: initially assuring betweeness.

The teacher's closing remark [53T] introduced a new immediate-object, being, due to the random position of $X$, equidistance cannot be deduced from its betweeness relationship with $A$ and $B$. The teacher's sign-interpretant probably contains his knowledge that the BetweenPoint-Theorem guarantees the existence of a point with a nonspecific position, contrary to what is required for the midpoint. It might include his supposition that María comprehends the mistake Laura and Ángela are making, and his realization of the opportunity to explain why RCFP is an incorrect procedure in this situation. That is why the third didactical-dynamicobject (Tddo3) appears to be the following: it is necessary to invalidate starting the proof of the Midpoint-Existence-Theorem guaranteeing betweeness, something the students might have noticed through his representation of possible positions for the point.

Moment 4: The point should be created with a different condition. The teacher explicitly stated the invalidity of RCFP (immediate-object): "And do we want $\boldsymbol{X}$ to appear somewhere over there or do we want $X$ to be very special? Where do we want $X$ to appear? In graphic terms, only about here (he signals the middle of the segment), right? So $X$ is actually the midpoint. If we do as they suggest [referring to Laura and Ángela], you are going to force the poor point, which has been born already, to satisfy conditions. Remember what I said yesterday? That it is better when the point is born..." [53T]. His unfinished sentence was completed by students "....it is born with the conditions", which he picked up, "It should be born with the conditions we want. Do you see how different the matter is? Is it clear why the given suggestion doesn't work out?" [54T]. The students responded agreement. 
The above dialogue provides evidence to infer that the teacher's previous sign-interpretant did include his belief that the students lacked understanding the logical difficulty that is generated due to RCFP. He probably thought they comprehend why using the PointBetween-Theorem gives rise to RCFP. Therefore, he wanted to focus on the correct way to start the proof, which is part of his current sign-interpretant. His didactical-dynamic-object (Tddo4) is the necessity that students understand the invalidity of RCFP, which he believes will be attained via the born metaphor he uses.

Moment 5: Is it "legal" to consider a point with exactly the coordinate we need? The teacher's next sign-vehicle includes the representation on the blackboard of the special point on the segment. To favor a change of track in the students' thinking, he referred to a construction: "At some moment we shall need betweeness, but not now. Rather, we want betweeness to be the result of another construction that we must do. [...] So, how can we construct this little point $X$ [he pointed to what could be the midpoint of the segment represented on the blackboard and erased the other marked points] so that it is born with the conditions we want it to have at birth? What do you think we can do?" [57T]. Juan's idea was to assign coordinates to the endpoints of the segment [58J]. Dina, expressing doubt because she considers that this could be too strong an assumption, wanted to assign equidistance, $A X=X B$. She asks if this will be "legal" ${ }^{10}$ [60D, 62D, 64D].

The teacher interpreted that the students understood why the Between-Point-Theorem cannot be used to generate the midpoint; he knew that the proof requires using the LineReal Numbers-Postulate to assign coordinates to the segment's endpoints, and should start with the creation of a point satisfying the equidistance property because betweeness will be a direct consequence of it (sign-interpretant). Having discarded Laura's and Ángela's proposal, the teacher asked for ideas to create a point that satisfies equidistance to the endpoints of the segment (immediate-object). The reference to a construction leads us to believe that his didactical dynamic-object (Tddo5) is now the identification of the necessity to give coordinates to the endpoints of the segment which becomes the means to find the midpoint as the point whose coordinate is their average.

The students' sign-interpretant includes the conception of the midpoint as a point that is equidistant from the endpoints which can be expressed using coordinates. Their immediateobject is creating such a point, which coincides with the teacher's immediate-object. Dina's dynamic-object ( $\mathrm{S} d o 4)$ is the uncertainty on the validity of choosing coordinates to assure equidistance.

Moment 6: Equidistance using coordinates. The teacher accepted Dina's idea with his following sign-vehicle: "Okay. We are going to see if it is "legal" or not. In essence, what you are thinking of is to assign coordinates to the points $A$ and $B$ (pointing at the figure on the blackboard). Using these coordinates, what do we do?" [62T]. It is Ángela who answered: "That the coordinate of $X \ldots$ then that the distance $A X$ be equal to the distance $X B$. Since we already have coordinates, then make it have the coordinate... that is, the distance between the coordinates... or between the points be equal. That is, the distance $A X$ be equal to the distance $X B$ " [68-70-72Á]. The teacher completed his sign-vehicle: "Okay. Ángela has said a very important thing. Dina's idea of giving $A$ and $B$ coordinates is good, and then look for the coordinate of $X$ so that $A X$ is equal to $B X$." [71T].

\footnotetext{
${ }^{10}$ The slang that Dina uses in Spanish (machetear) is replaced with the colloquial English use of "legal". It means using an incorrect or imprecise mathematical procedure which doesn't lead to an incorrect answer.
} 
The teacher's sign-interpretant is that the students now understand that equidistance will be attained by using coordinates for points $A$ and $B$. The teacher knows that the use of the LineReal Numbers-Postulate is required. The teacher's didactical dynamic-object (Tddo6) is: the students need to understand how to use the coordinates and the theoretical warrant. His immediate-object is approval of the method proposed to find $X$.

Ángela approved and contributed to this different way of creating $X$. Her dynamic-object could be approaching the teacher's immediate-object, or she is simply repeating what others have already said without really understanding why it's the correct way to proceed. Her immediate-object is the outline of the way to find $X$ via coordinates so its distance to $A$ and to $B$ is equal. It's possible that all the students' sign-interpretant is the general idea of the use of coordinates without a clear way of how to operate with them.

What followed in the episode was the construction of the point $X$ with the required property, assigning $z$ and $y$ as coordinates of $A$ and $B$, respectively. The students had a hard time creating the real number $w$ that should be the coordinate of $X$ to assure equidistance to $A$ and $B$; they realized that the theoretical element they used was the Line-Real Numbers-Postulate.

\subsection{Teacher semiotic mediation}

The analysis of the episode allows us to examine, in depth, the teacher's semiotic mediation which can be outlined as follows. The teacher was aware that the first step proposed is not the one required, but he allowed Laura and Ángela to develop their idea, the construction of a point $M$ such that $A-M-B$ (Sdo1). He solicited a complete argument and also an informal explanation of the idea, hoping they would realize that the Point-Between-Theorem does not provide the elements necessary to prove the existence of the midpoint (Tddo1). The students remained firm in their initial proposal, even trying to present it in a "more formal" way (Sdo2). This led the teacher to resort to other visual and graphical strategies to help students realize that equidistance cannot be deduced from betweeness.

He asked the students to recall the midpoint definition and represented the situation graphically (Tddo2). Laura and Ángela did not grasp the lack of validity of their proposal even though the teacher's continuous insistence to revise it implicitly conveyed that idea. He realized the need to invalidate the use of the Point-Between-Theorem as a starting point for the proof (Tddo3) because the students were going to recur to RCFP. María expressed her understanding that choosing a point "freely" means other properties cannot be forced on it, which would happen if they continued with Laura's and Ángela's proposal (Sdo3). The teacher then focused on the necessity of having the students understand why RCFP is not a correct procedure (Tddo4). Once he thought they had understood the invalidity of RCFP, the teacher's mediation shifted to promote student meaning-making of the use of coordinates to construct point $X$ (Tddo5). Eventually, the students proposed this idea though doubting whether it was the correct way to proceed (Sdo4). The teacher wasn't sure the students knew how to use the coordinates of the endpoints of the segment for their endeavor, and the role that the Line-Real Numbers-Postulate would play (Tddo6), although they expressed the general idea (Sdo5).

The following figure (Fig. 1) maps out the emergence and interleaving of teacher and student dynamic sign-objects. Without the teacher's awareness of students' own interpretations and his open acknowledgment of their existence, and without the students' effort to actively involve themselves in their own understanding, the semiotic mediation of the teacher would have been impossible. 


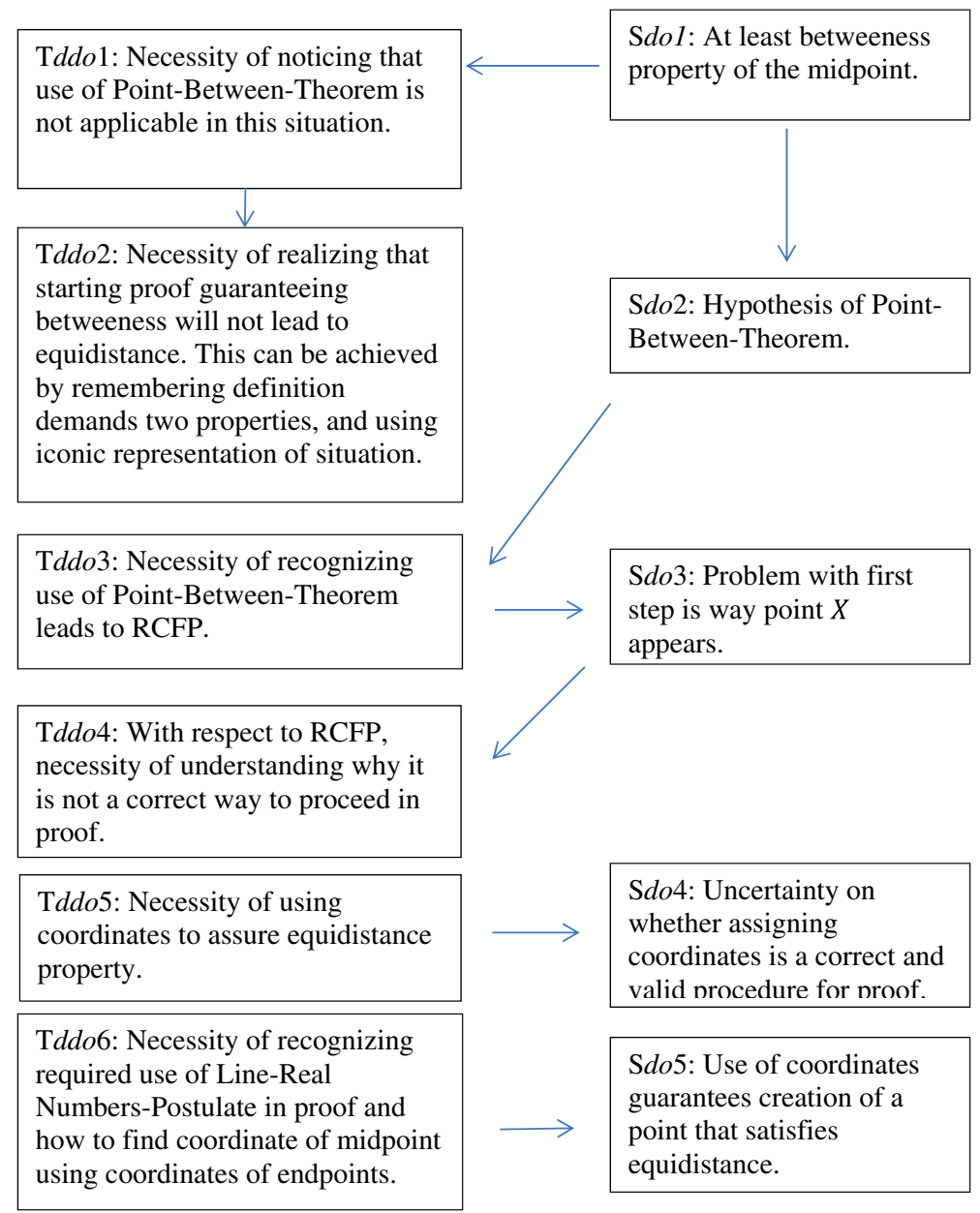

Fig. 1 Outline of teacher semiotic mediation

\section{Final considerations}

We have observed that the incorrect procedure RCFP is frequently proposed by students when they have to prove the existence of a special point or ray. This phenomenon is probably not perceived when teaching and is only centered on the teacher's presentation of proofs and the students' passive recording, or when they just read textbook proofs. However, this issue is very noticeable when the pedagogical strategy of the teachinglearning of geometry is based on the conformation of a theoretic system as product of joint teacher-student mathematical activity such as empirically exploring geometric situations to formulate conjectures that give rise to theorems, definitions and postulates, as is the norm in this course. Although the students knew the proof should start by constructing a point, their decision of simply choosing a point between the endpoints of the segment lead to RCFP which is always an incorrect procedure. They did not realize 
that whatever condition they initially put on the point must cause, in a direct way, the other required properties. The teacher's main role throughout the proof construction process was to query students' proposals, letting them autonomously suggest the proof steps; not guiding them. This is why the teacher foresaw RCFP, and recognized that letting the students develop their idea was an opportunity to promote meaning-making of the unacceptability of the procedure.

Two issues can be drawn from this analysis. (1) Do the students understand that the first step for an existence proof is correct depending on whether the other required conditions can be deduced from those first assumed? (2) What is causing the students to spontaneously propose creating a random object that leads them to force wanted properties on it (RCFP)? Dina's last reported statement can give us some insight on this second issue. The core of her question seems to be whether or not allowing the point to have such a strong property, equidistance from the endpoints, is "legal". RCFP seems to be a natural strategy for the students because it is proposed again when proving other existence theorems: Point-Localization $^{11}$ and Angle-Bisector-Existence.

We think that the core of the problem is the cognitive dilemma that arises when constructing objects that share properties with those whose existence must be proven. Students probably ask themselves: why not construct them with all the properties from the beginning? How does one know which and how many of the properties should be assigned initially? The answer to this question needs the teacher's didactical guidance. That is, when the course is conducted under the belief that learning means comprehensively adopting historically established ways of acting, and modifying and extending one's discourse about mathematical objects and procedures (Ben-Zvi \& Sfard, 2007), then understanding the procedure to prove the existence of a mathematical object defined by more than one property should be done under the teacher's direct and explicit guidance. There is no other way of knowing all of this without "an interaction with a competent doer" (Ben-Zvi \& Sfard 2007, p. 118). The teacher should have explicitly analyzed, from the mathematical point of view, the acceptability of assigning a strong condition when creating the object, if it can be justified. A teacher cannot expect students to discover how to proceed autonomously in these cases.

The other issue that may cause the students' cognitive dilemma of how to begin the proof has to do with the logic behind a conjunction. Conjunctions are commutative statements, yet setting one or the other property initially makes a difference, as is the case when proving the existence of the midpoint. How to understand something that seems to contradict commutativity? Even more conflicting is the fact that part of the thesis of the conditional statement to be proved is established at the beginning, because these are usually the last steps in the proving process. The proofs students had been constructing were developed by connecting arguments that are deduced from previous ones; these did not include the introduction of an object with some of the properties mentioned in the thesis of the statement. There is another possible cause with respect to theorems in which the thesis is a conjunction. Do the students think that the order in which the properties are mentioned indicates the order in which each one has to be assured?

Since the framework we adopted centers on interpretation, we had to be conscious of not only, as is usual, the successful conveying of ideas but also of communication that was not effective. We had to study the possible student sign-interpretants, not just the sign-vehicles, to be able to deduce what aspect of the $R M O$ they were considering. We had to look beyond the

$\overline{{ }^{11} \text { If } r \text { is a positive real number and }} \overrightarrow{C T}$ any given ray, then there exists a point $X \in \overrightarrow{C T}$ such that $C X=r$ 
specific sign-vehicle and consider a complete semiosis cycle to determine possible interpretants. Thus the aspects that are generally disregarded, those that apparently have no meaning or are erroneous, gained relevance.

We believe this paper contributes to the model for assessing student proof comprehension, proposed by Mejía-Ramos, Fuller, Weber, Rhoads, and Samkoff (2012). Their model is directed towards evaluating students' understanding of two main features of a proof they have read: mathematical aspects of the statements involved in the proof (status, meaning of terms, warrants) and aspects of the proof as a mathematical entity (structure, scope, applications). Yet, understanding a proof also includes realizing why a specific way of proceeding to establish the thesis is not mathematically correct, as is the case of RCFP.

Acknowledgments Our long-term project was financed by the Colombian national research institute, Colciencias, and Centro de Investigaciones, Universidad Pedagógica Nacional (CIUP). The views and conclusions expressed here are those of the authors and not of the institutions.

We want to thank the reviewers and editor for their suggestions which definitely improved this article.

\section{References}

Baccaglini-Frank, A., \& Mariotti, M. A. (2010). Generating conjectures in dynamic geometry: The maintaining dragging model. International Journal of Computers for Mathematical Learning, 15, 225-253.

Ben-Zvi, D., \& Sfard, A. (2007). Ariadne's thread, Daedalus' wings, and the learner's autonomy. Education \& Didactique, 1(3), 117-134.

Birkhoff, G. (1932). A set of postulates for plane geometry, based on scale and protractor. Annals of Mathematics, 33(2), 329-345. Retrieved from in http://www.jstor.org/stable/1968336.

de Guzmán, M., Hodgson, B., Robert, A., \& Villani, V. (1998). Difficulties in the passage from secondary to tertiary education. Documenta Mathematica (extra volume). Proceedings of the International Congress of Mathematicians (Berlin), 3, 747-762.

Lannin, J., Barker, D., \& Townsend, B. (2007). How students view the general nature of their errors. Educational Studies in Mathematics, 66, 43-59.

Mariotti, M. A. (2009). Artifacts and signs after a Vygotskian perspective: The role of the teacher. ZDM, 41(4), $427-440$.

Mejía-Ramos, J. P., Fuller, E., Weber, K., Rhoads, K., \& Samkoff, A. (2012). An assessment model for proof comprehension in undergraduate mathematics. Educational Studies in Mathematics, 79(1), 3-18.

Perry, P., Camargo, L., Samper, C., \& Rojas, C. (2006). Actividad demostrativa en la formación inicial del profesor de matemáticas [Proving in pre-service mathematics teachers' training]. Bogotá: Fondo Editorial de la Universidad Pedagógica Nacional.

Perry, P., Samper, C., Camargo, L., \& Molina, Ó. (2013). Innovación en un aula de geometría de nivel universitario [Innovation in a university geometry classroom]. In C. Samper \& Ó. Molina. Geometría Plana: un espacio de aprendizaje [Plane Geometry: A setting for learning]. Bogotá: Fondo Editorial de la Universidad Pedagógica Nacional.

Perry, P., Camargo, L., Samper, C., Sáenz-Ludlow, A., \& Molina, Ó. (2014). Teacher semiotic mediation and student meaning-making: A Peircean perspective. In P. Liljedahl, S. Oesterle, C. Nicol, \& D. Allan (Eds.), Proceedings of the 38th Conference of the International Group for the Psychology of Mathematics Education and the 36th Conference of the North American Chapter of the Psychology of Mathematics Education (Vol. 4, pp. 409-416). Vancouver: PME.

Radford, L., Schubring, G., \& Seeger, F. (2011). Signifying and meaning-making in mathematical thinking, teaching, and learning. Educational Studies in Mathematics, 77(2-3), 149-156.

Sáenz-Ludlow, A., \& Zellweger, S. (2012). The teaching-learning of mathematics as a double process of intraand inter-interpretation: A Peircean perspective. In Pre-proceedings of the 12th ICME. Retrieved from http:// www.icme12.org/data/ICME12_Pre-proceedings.zip.

Samper, C., Camargo, L., Molina, Ó., \& Perry, P. (2013). Instrumented activity and semiotic mediation: Two frames to describe the conjecture construction process as curricular organizer. In A. M. Lindmeier \& A. 
Heinze (Eds.), Proceedings of the 37th Conference of the International Group for the Psychology of Mathematics Education (Vol. 4, pp. 145-152). Kiel: PME.

Selden, A., \& Selden, J. (2011). Mathematical and non-mathematical university students' proving difficulties. In L. R. Wiest \& T. D. Lamburg (Eds.), Proceedings of the 33rd Annual Conference of the North American Chapter of the International Group for the Psychology of Mathematics Education (pp. 675-683). Reno: University of Nevada.

Weber, K. (2001). Student difficulty in constructing proofs: The need for strategic knowledge. Educational Studies in Mathematics, 48(1), 101-119. 